\title{
Sciatic Nerve Regeneration in Wistar Albino Rats Evaluated by in vivo Conductivity and in vitro $1 \mathrm{H}$ NMR Relaxometry
}

\author{
Anamaria Victoria Bumbu1*, Radu Fechete², Marcel Perian³, Bogdan Septimiu Bumbu³, Klara \\ Brinzaniuc $^{3}$ \\ 1. Spitalul Clinic Judetean De Urgenta Oradea, Romania \\ 2. Technical University of Cluj-Napoca, Romania \\ 3. University of Medicine and Pharmacy Tîrgu Mures, Romania
}

Objective: The aim of this study was to evaluate and quantify functional and structural nerve regeneration after reconstruction using either direct suture or silicon graft. Methods: Thirty-two adult Wistar Albino rats were divided in two equal groups. The left sciatic nerve was crosssectioned and reconstructed using either direct suture (DS group) or a silicone graft (SG group). At 4, 6, 8 and 10 weeks two rats were randomly chosen from each group for in vivo measurement of nerve electric conductivity and subsequently sacrificed together with other two rats from the same group for in vitro $1 \mathrm{H}$ NMR relaxometry measurements. The T2 distributions were assigned to $1 \mathrm{H}$ located in different pools corresponding to the nerve structure. Results: In the injured nerve we observed a significant increase in the stimulation threshold and a decrease in conduction velocity when compared with the healthy nerve in both groups. Whereas the conduction velocity increased progressively from 4 to 10 weeks in the DS group, the opposite evolution was observed in the SG group. In both groups, the first two peaks corresponding to water bound to collagen and epineurium had smaller transverse relaxation times in the injured nerves, while there was no change in the peaks corresponding to perineurium and free water between healthy and injured nerves. Conclusions: Significant differences were observed between direct suture and nerve graft reconstructions from both a functional and structural point of view. In the case of direct suture reconstruction, the nerve was functionally healed at 10 weeks after injury.

Keywords: rat's sciatic nerve regeneration, electric conductivity, $1 \mathrm{H}$ NMR relaxometry

Received 8 November 2017 / Accepted 15 January 2018

\section{Introduction}

In the last twenty years, the treatment methods regarding patients with a peripheral nerve injury has significantly evolved. Due to better understanding of the structure and function of the peripheral nerves, new intraoperative diagnosis, reconstructive surgery is being optimized permanently. Peripheral nerves are designed to receive and relay input from the environment to the central nervous system. They are associated with three separate tissue sheaths, the endoneurium, perineurium and epineurium. These three elements have been investigated over time and it has been demonstrated that they are providing multiple mechanical and physiologic functions [1].

The retrograde cell death of motor and sensory neurons is one of the main problems associated with peripheral nerve injury. Compared to the central nervous system, injured peripheral axons are able to self-regenerate. Sometimes misdirected growth of nerve fibres can occur, even if the axonal regeneration across the injury site was successful. In most cases of peripheral nerve injuries, the nerve ends can be sutured, and the neurological recovery can be reasonable. However, if we have a nerve defect, the primary suture is not possible and nerve grafts are required to bridge the defect [2].
In vivo specific methods of examination of peripheral nervous system are based on electrodiagnosis and nuclear magnetic resonance imaging or spectroscopy. Electrodiagnosis is the observation and interpretation of the electrical signals derived from the depolarization and repolarization of the peripheral nerves. Voluntary muscle contractions can produce these action potentials, or they can be evoked by electrical stimulation [1]. In the early 90s, Howe et al [3] and Filler [4] proposed an MRI (magnetic resonance imaging) examination, called neurography, focused on the visualization of the peripheral nervous system with dedicated sequences optimizing the contrast between nerves and their environment. The neuronal ultrastructure is examined by a phased-array coils into a high field imager significantly improved the spatial resolution and the signal to noise ratio into an MR image. Such MR picture is spatially encoded by a specific NMR (nuclear magnetic resonance) parameter called, transverse relaxation time, $T_{2}$ which is specific to each tissue. The $T_{2}$ - weighted spin-echo sequences, have good contrast resolution, but they have been replaced by the neurography sequences which are highly weighted $T_{2}$ sequences using long echo times combined with fat signal suppression. MRI findings are consistent with the peripheral nerve`s anatomy [5]. Only few studies were reported on the degeneration/injury of the peripheral nerve on animals. Thus, Does and Snyder use the multi- 
exponential $T_{2}$ relaxation distribution on the amphibian Xenopus laevis of which sciatic nerve was crushed [6]. Recently the ${ }^{1} \mathrm{H}$ NMR relaxometry method combined with inverse Laplace analysis $[7,8]$ was successfully used to assess the ovariectomy induced osteoporosis on Wistar Albino rats [9-12]. The measured $T_{2}$-distribution on different section of rats femur allowed the calculation of the pores sizes distributions as well as the effects of treatment with simvastatin or fenofibrate.

The purpose of this study was to evaluate the Wistar Albino rats' sciatic nerve regeneration after injury through in vivo electrodiagnosis and in vitro ${ }^{1} \mathrm{H}$ NMR relaxometry.

\section{Methods}

The Ethics Committee of The University of Medicine and Pharmacy of Targu Mures approved the experiment and study protocols, according to document 45/2017. For this study we have used thirty-two Wistar Albino rats, with an average weight of 500 grams. The animals were divided into two groups, each having sixteen rats. For all animals the left sciatic nerve was interrupted using a No. 11 blade (Fig. 1a). In the first group the nerve was directly sutured under magnification loupes (2.5X Keeler) with 8.0 sutures (Fig. 1b). The left sciatic nerve of rats belonging to the second group, by interruption, a $7 \mathrm{~mm}$ defect was created and the reconstruction was performed using a silicone graft. The proximal and distal nerve stumps were inserted $1 \mathrm{~mm}$ into the tube to leave a $5 \mathrm{~mm}$ gap and they were fixed to the graft with two epineural sutures (8.0 Prolene) (see Fig. 1c). The right sciatic nerve for both groups was used as the control group.

In vivo electric conductivity measurements (Fig. 2) were performed for two randomly selected rats at 4, 6, 8 and 10 weeks after surgery. Electrical nerve stimulation was carried on using an external programmable pulse generator based on a PIC16F877 microcontroller produced by Microchip. Inc. , Arizona USA. The rectangular pulses were delivered with a period of 1 second, a $1 \mathrm{~ms}$ the pulse-width, having an amplitude of $2 \times$ threshold for each nerve. The stimulation electrode was bipolar type, using two parallel pure silver wires at $2 \mathrm{~mm}$ distance, placed approximately $1 \mathrm{~cm}$ proximal to the lesion.

The nervous influx was recorded using bipolar silver electrodes placed distal to the lesion, amplified using an AD621 instrumentation amplifier produced by Analog Devices and fed into a National Instruments acquisition board. LabView software was used to view, store and analyze the recorded signals. Using a digital vernier calliper, we measured the distance between the stimulation and the receiving electrode. The nerve conduction time was measured in LabView and the conduction speed was calculated. Ten measurements of distance, conduction time and stimulus amplitude were performed for each nerve, and the average values were reported.

At 4, 6, 8 and 10 weeks after the injury and reconstruction, 4 animals from each group were randomly selected and euthanized with an overdose of ketamine and xiline $(8-10 \mathrm{mg} / \mathrm{kg})$. Both sciatic nerves were removed and preserved into a solution of formaldehyde until the NMR
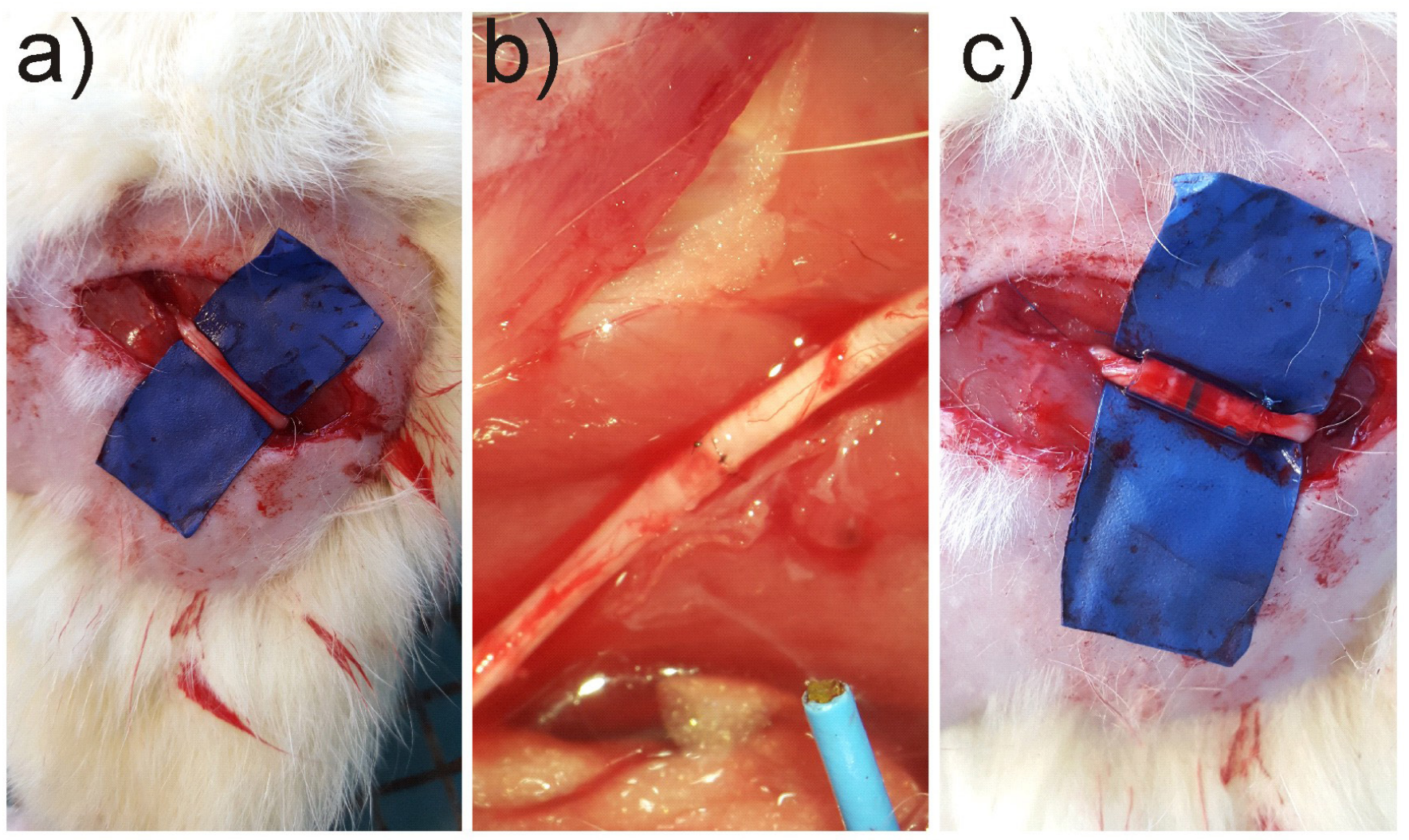

Fig. 1. Picture of a) healthy nerve; b) direct suture method and c) silicone nerve graft method for Wistar Albino rats. 


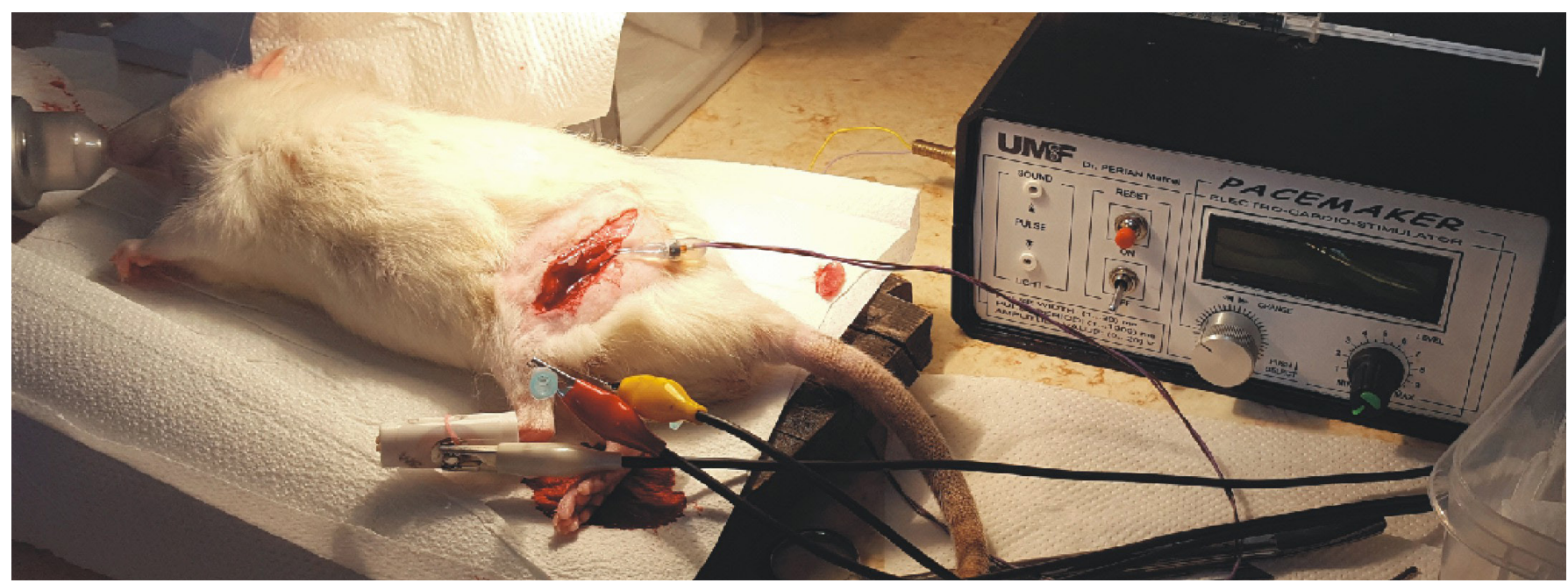

Fig. 2. Picture of the experimental set-up for the in vivo electric conductivity measurement of rat's sciatic nerve.

measurements were performed. The low field NMR measurements were performed using the BRUKER MINISPEC mq20 spectrometer working at $19.6712 \mathrm{MHz}$. The interecho time in CPMG pulse sequence was set at $0.25 \mathrm{~ms}$. The tipping pulse length was $9 \mu$ s and refocusing pulses with the same duration was set double in amplitude. A total number of 3000 echoes were recorded (see Fig. 3a). The decay of transverse magnetization during the CPMG echoes train was assumed to be multi-exponential and hence can be described by equation depicted in Fig. 3. Here $T_{2}$ is the transverse relaxation time of each particular statistical sub-ensemble of protons and $f\left(T_{2}\right)$ is the distribution function. The interpretation of $1 \mathrm{D}$ experimental data request to find the transverse relaxation time distribution $f\left(T_{2}\right)$. To this purpose the experimental data was analyzed using a Laplace inversion algorithm. The mathematically details were detailed elsewhere $[7,8]$. An example of such $T_{2}$-distribution is presented in Fig $3 \mathrm{~b}$ for the injured sciatic nerve of the rat No. 8 sacrificed at 6 weeks after direct suture (group DS). Usually, the association of each distinct peak with specific hydrogen reservoirs (mostly water) is based on the water molecule mobility, then the most mobile ${ }^{1} \mathrm{H}$ are characterized by a large transverse relaxation time, $T_{2}$ while the most rigid ${ }^{1} \mathrm{H}$ are characterized by the smallest $T_{2}$.

\section{Results}

The average values of response voltage, response time and response velocity are presented in Fig. 4 for both groups (direct suture and silicone graft reconstruction methods) function of time elapsed from reconstruction. In each case parameters measured for the healthy nerve (red bars) are compared with those measured for injured sciatic nerve (blue bars).

A clear differentiation can be observed between the healthy nerve's average voltage response $(2.8-4.2 \mathrm{~V})$ and under-recovery sciatic nerve with the corresponding values between $4-6.5 \mathrm{~V}$ (see Fig. 4a). The net differentiation is

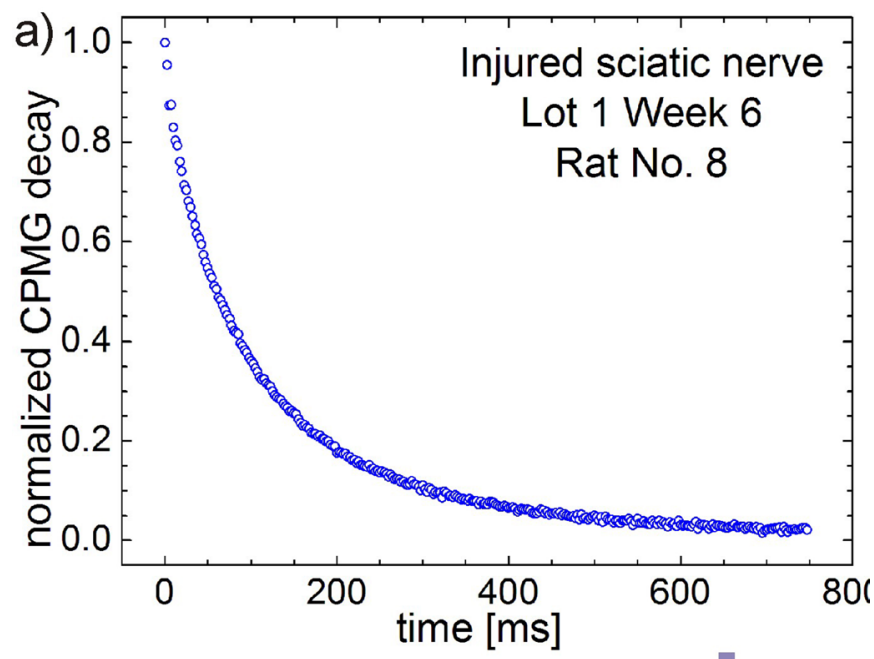

inverse Laplace transform

$$
M_{C P M G}(t)=\int_{0}^{\infty} f\left(T_{2}\right) e^{-\frac{t}{T_{2}}} d T_{2}
$$

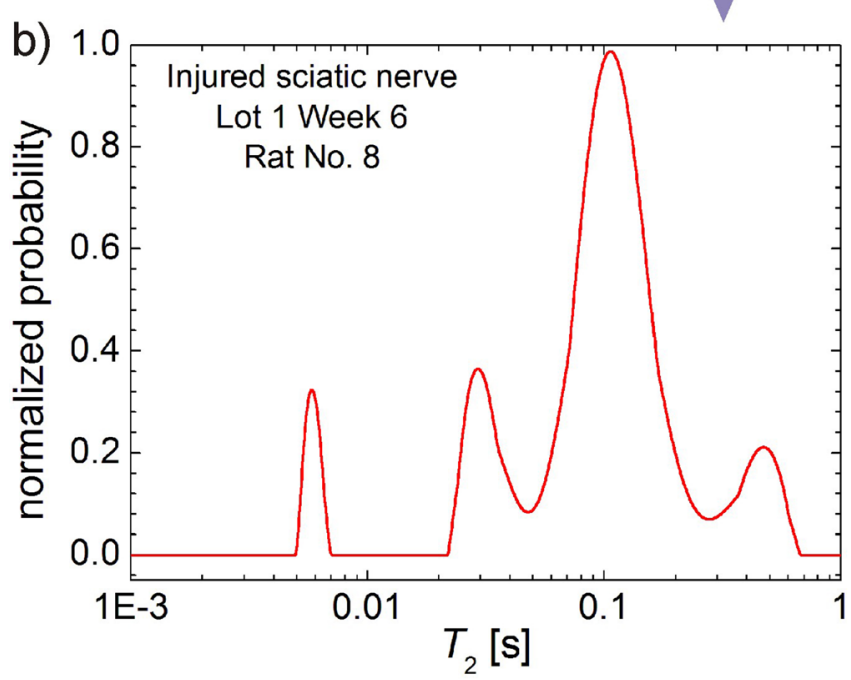

Fig. 3. a) The normalized CPMG decay measured after 6 weeks from injury and direct suture reconstruction of Rat's No. 8 sciatic nerve and b) the corresponding T2-distribution obtained by inverse Laplace transform. 

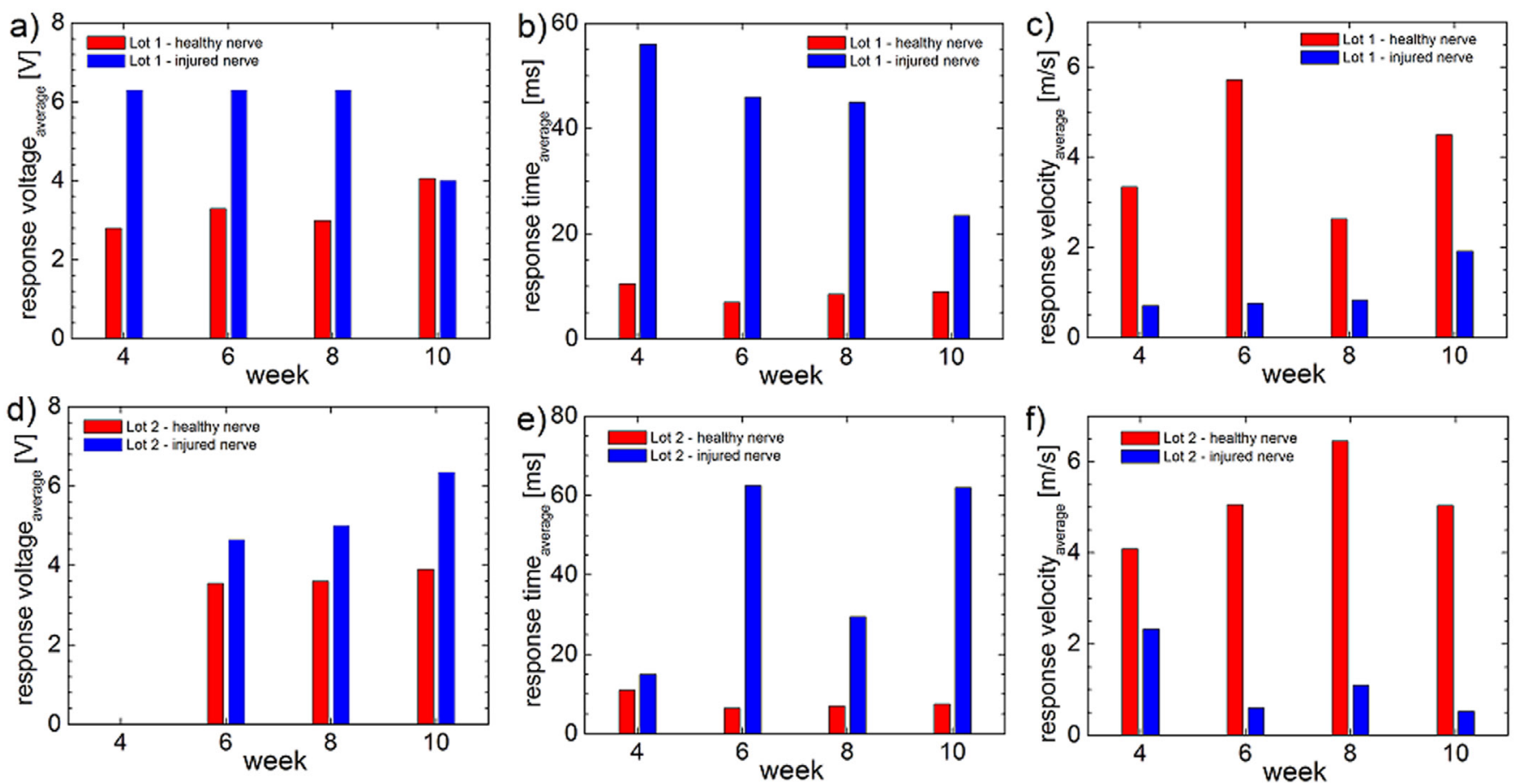

Fig. 4. In vivo electric conductivity comparison between the direct sutured group - a, b, c; and the silicon grafted group - d, e, f. Measured parameters were average voltage response $-a$, $d$; time response $-b, e$; and velocity response $-c, f$.

observed for the sciatic nerve measured at an early and medium recovery time ( $4-8$ weeks after injury and suture). At 10 weeks after injury the average voltage response values are the same for injured and healthy nerve. The average time response for the healthy nerve is much shorter $(<11$ ms) compared with the same average time measured for the injured and directly sutured sciatic nerve (>23 ms) (Fig. $4 \mathrm{~b})$. A fast response time is associated with a large average conduction velocity (see Fig. 4c). The large difference between the measured values characterizing the in vivo electric conduction of the healthy and injured sciatic nerve of the group DS, overcome the small fluctuations of the corresponding values measured in time for the healthy nerve (see the left column of Fig. 4).

The healing behavior, as resulted from in vivo electric conductivity measurement on Wistar Albino rats from group SG was unexpected. Large differences were observed for all three parameters, the average voltage, time and velocity, measured at 10 weeks after surgery (see the right column in Fig. 4). In fact it appears that, for this group, the sciatic nerve functionality is decaying while time passes, since smaller differences between measured parameters are observed at an early healing process. Due to the fluctuation of values along the recovery time, the average response time and velocity appears not to be able to describe satisfactorily the healing process (see the blue bars in Fig. $4 \mathrm{e}$ and $4 \mathrm{f}$ ).

The electric conductivity measurement is an expression of the nerve function while the ${ }^{1} \mathrm{H}$ NMR relaxometry measurement can describe the regeneration process from a structural point of view. The average normalized $T_{2}$-distributions measured for the healthy and recovering after injury and direct suture Wistar Albino rats' sciatic nerves (group DS) are presented in Fig. 5 for rats sacrificed at 4,
6, 8 and 10 weeks. All $T_{2}$-distributions presents four peeks associated with characteristic protons' mobility to i) ${ }^{1} \mathrm{H}$ bounded to collagen fibrils characterized by the smallest $T_{2}$ values between $1.35 \mathrm{~ms}-11.2 \mathrm{~ms}$ for the healthy nerve (Fig. 5a) and between $1.19 \mathrm{~ms}-7.09 \mathrm{~ms}$ for the injured nerve (Fig. 5b); ii) ${ }^{1} \mathrm{H}$ from water located in epineurium characterized by $T_{2}$ values between $7.64 \mathrm{~ms}-48.89 \mathrm{~ms}$ for the healthy nerve and between $7.00 \mathrm{~ms}-45.37 \mathrm{~ms}$ for the injured nerve; iii) ${ }^{1} \mathrm{H}$ from water located in perineurium characterized by $T_{2}$ values between $25.7 \mathrm{~ms}-266.2 \mathrm{~ms}$ for the healthy nerve and between $23.3 \mathrm{~ms}-410.0 \mathrm{~ms}$ for the injured nerve; iv) ${ }^{1} \mathrm{H}$ from water from blood vessels characterized by $T_{2}$ values between $139.0 \mathrm{~ms}-926.6 \mathrm{~ms}$ for the healthy nerve and between $284 \mathrm{~ms}-1160 \mathrm{~ms}$ for the injured nerve. At 6 weeks the peaks appear to be better resolved while at 8 weeks after surgery the peaks characterized by the larger $T_{2}$ value merges with the main peak.

The average normalized $T_{2}$-distributions measured for the healthy and recovering after injury and reconstruction of sciatic nerves with silicone graft (group SG) are presented in Fig. 6. As in the previous case all $T_{2}$-distributions present the same four peeks with the same association. Then, the ${ }^{1} \mathrm{H}$ bounded to collagen fibrils presents $T_{2}$ values between $1.91 \mathrm{~ms}-8.5 \mathrm{~ms}$ for the healthy nerve (Fig. 6a) and between $0.1 \mathrm{~ms}-4.19 \mathrm{~ms}$ for the injured nerve (Fig. 6b). The ${ }^{1} \mathrm{H}$ from water located in epineurium are characterized by $T_{2}$ values between $6.72 \mathrm{~ms}-46.37 \mathrm{~ms}$ for the healthy nerve and between $5.08 \mathrm{~ms}-41.2 \mathrm{~ms}$ for the injured nerve. The ${ }^{1} \mathrm{H}$ from water located in perineurium have $T_{2}$ values between $27.41 \mathrm{~ms}-261.33 \mathrm{~ms}$ for the healthy nerve and between $20.91 \mathrm{~ms}-466.0 \mathrm{~ms}$ for the injured nerve; Finally, the ${ }^{1} \mathrm{H}$ from water from blood vessels is characterized by $T_{2}$ values between $143 \mathrm{~ms}-816$ 

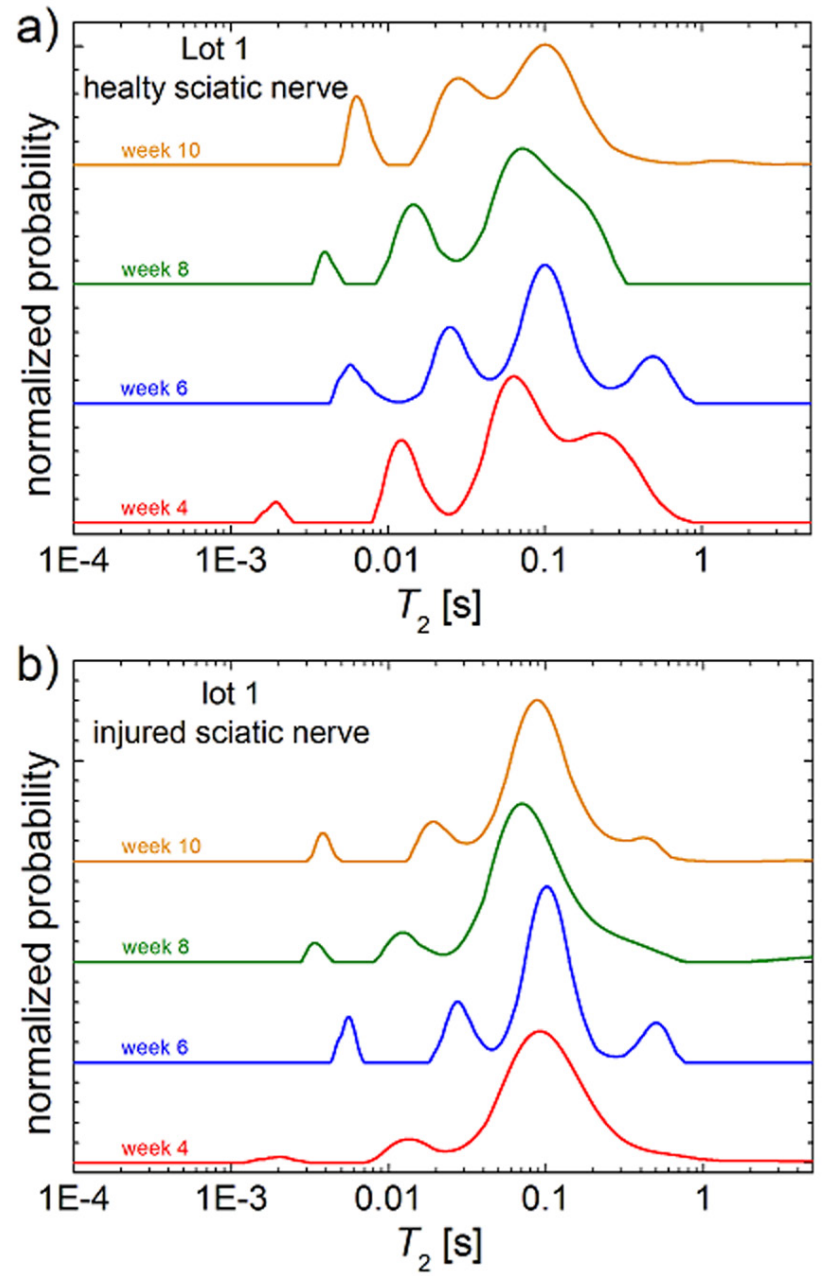

Fig. 5. Compared normalized T2 - distributions measured for the rat's sciatic nerve a) healthy and b) at 4, 6, 8 and 10 weeks after direct suture.

ms for the healthy nerve and between $276 \mathrm{~ms}-1185 \mathrm{~ms}$ for the injured nerve. As in the case of the group DS, in group SG the $T_{2}$-distributions presents broaden peaks at 8 weeks after surgery.

\section{Discussion}

The response voltage is an important parameter which can be associated with the healthy state of the sciatic nerve. Thus, a healthy nerve will respond (carrying the electric signal) to a lower stimulating voltage.

The in vivo electric conductivity measurement for group DS presents a clear recovery after injury and direct sciatic nerve reconstruction. Therefore, form the average voltage response point of view, the rat's sciatic nerve is healed at 10 weeks after injury. Contrary, from the average time response and consequently from average velocity response, the rats' sciatic nerve is not healed therefore the nerve function is not completely restored. Nevertheless, the overall behavior of the electric conduction parameters show that the nerve is in the healing process since these values become closer to the corresponding values measured for the healthy nerve. Moreover, the recovering nerve (left one) presents a more predictable behavior than the more fluctuating behavior observed for the healthy nerve (the right
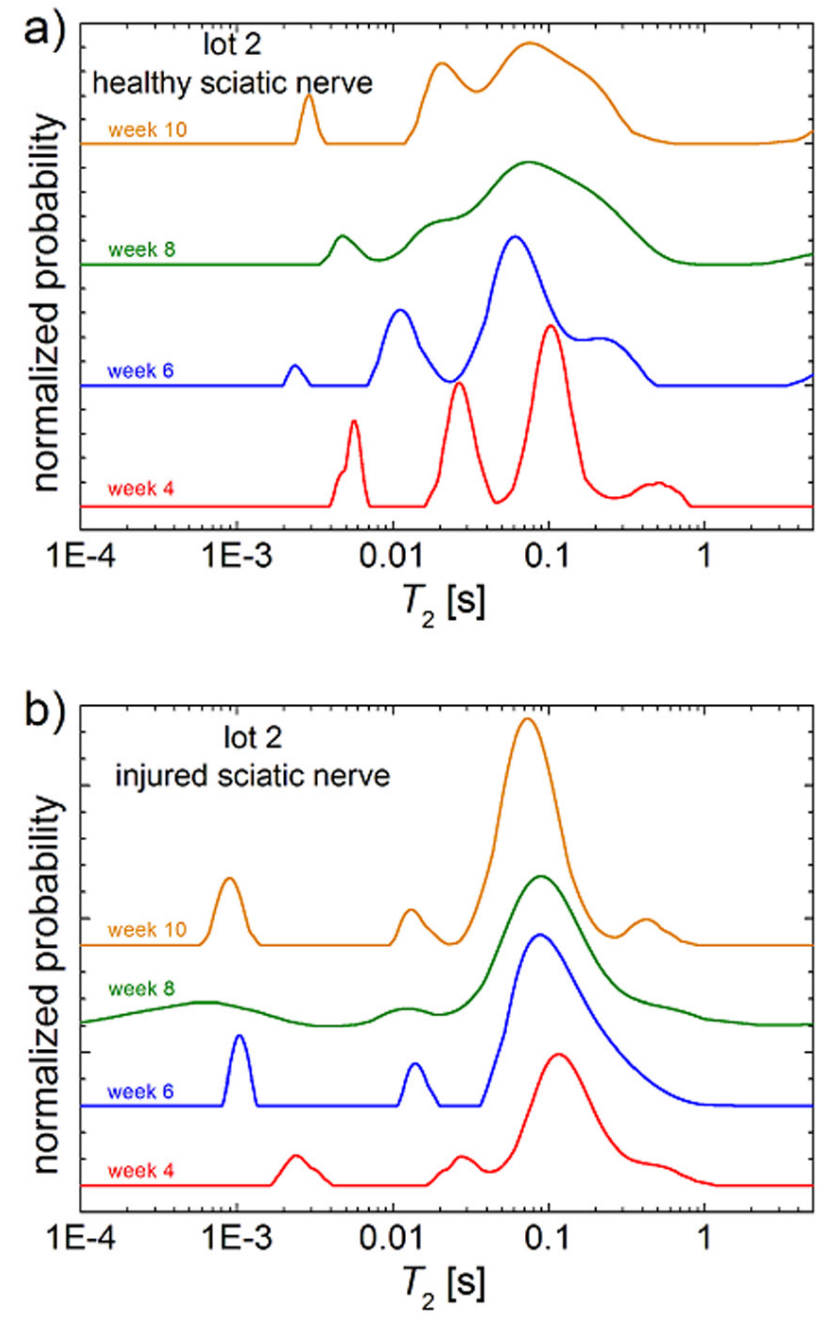

Fig. 6. Compared normalized T2 - distributions measured for the rat's sciatic nerve a) healthy and b) at 4, 6, 8 and 10 weeks after silicone grafting.

one of the same rat). The sudden change in the values of all three measured electric conduction parameters between the week 8 and week 10 can be explained by the healing process which takes place from proximal to distal part of the nerve [1]. One can consider, that until the $8^{\text {th }}$ week, the healing process did not reach the injury site while sometime after 8 weeks the process passes over the lesion and the functionality of the sciatic nerve is faster restored.

As a general observation the $T_{2}$-distributions measured for the injured sciatic nerve for both groups, presents a main peak (the majority of ${ }^{1} \mathrm{H}$ characterizing a certain pool) associated with water located in the perineurium. The evolution in time of this peak can lead to the interpretation of healing process in terms of structural changes at the nerve level. In all cases an interesting process was observed at 8 weeks which leads to a broadening of the $T_{2^{-}}$ distributions. It is possible to associate this phenomenon with the front of the healing process which is closer to the injured site.

\section{Conclusion}

In vivo electric conductibility and ${ }^{1} \mathrm{H}$ NMR relaxometry techniques are sensitive and can be used to evaluate the 
nerve healing process from direct point of view of functionality and structural changes. Significant differences were observed between the two types of reconstruction, direct suture or nerve graft. In this sense, the electric conductivity showed that the functionality of the sciatic nerve is restored faster, in the case of direct suture compared to the silicone graft. The ${ }^{1} \mathrm{H}$ NMR relaxometry and electric conductivity highlights the $8^{\text {th }}$ week, when we assumed that the frond of healing process is closer to the former injury site.

\section{Conflict of inerest}

None to declare.

\section{References}

1. Tenzis K. Julia, Smith L. Kevin - Repair and Grafting of the Peripheral Nerve. McCarthy G. Joseph, Plastic Surgery Volume 1 General Principles, W. B. Saunders Company, p;630-670.

2. Pettersson J., Kalbermatten D., McGrath A., Novikova L. Biodegradable fibrin conduit promotes long-term regeneration after peripheral nerve injury in adult rats. Journal of Plastic, Reconstructive \& Aesthetic Surgery. 2010;63:1893-1899.
3. Howe FA, Filler AG, Bell BA, Griffiths JR. - Magnetic resonance neurography. Magn Reson Med. 1992;28(2):328-38.

4. Filler AG, Kliot M, Winn HR, Tsuruda JS, Hayes CE, Howe FA, et al. Magnetic resonance neurography. Lancet 1993;341(8846):659-61.

5. Ohanaa, M., Moserb T., Moussaouïc A., et al. - Current and future imaging of the peripheral nervous system. Diagnostic and Interventional Imaging. 2014;95: 17-26.

6. Does M., Snyder R. - Multiexponential T2 Relaxation in Degenerating Peripheral Nerve. MRM. 1996;35:207-213.

7. Venkataramanan L., Song Y., Hurlimann M. - Solving Fredholm integrals of the first kind with tensor product structure in 2 and 2.5 dimensions. IEEE Trans Sign Proc. 2002;50:1017-1026.

8. Song Y., Venkataramanan L., Hürlimann M., et al. - J. Magn. Reson. 2002;154:261-268

9. Sipos R., Fechete R., Moldovan D., Sus I., Szasz S, Pávai Z. - Assessment of femoral bone osteoporosis in rats treated with simvastatin or fenofibrate. Open Life Sci. 2015;10:379-387.

10. Sipos R, Fechete R, Chelcea R, et al. - Ovariectomized rats' femur treated with fibrates and statins. Assessment of pore-size distribution by 1H-NMR relaxometry. Rom J Morphol Embryol. 2015;56 (2 Suppl):743-752

11. Chelcea R., Sipos R., Fechete R., et al. - One-Dimensional Laplace Spectroscopy Used For The Assessment Of Pore-Size Distribution On The Ovariectomized Rats Femur. STUDIA UBB CHEMIA. 2015; LX 1:57-70.

12. Sipos R., Fechete R., Moldovan D., et al. - Ovariectomy-Induced Osteoporosis Evaluated by $1 \mathrm{H}$ One-and Two-Dimensional NMR Transverse Relaxometry. Applied Magnetic Resonance. 2016;47 (12):1419-1437. 